\title{
Tunable Rigidity of (Polymeric Core)-(Lipid Shell) Nanoparticles for Regulated Cellular Uptake
}

\author{
Jiashu Sun, Lu Zhang, Jiuling Wang, Qiang Feng, Dingbin Liu, Qifang Yin, Dongyan Xu, \\ Yujie Wei, Baoquan Ding, Xinghua Shi,* and Xingyu Jiang**
}

Even though much research has shown that nanoparticles (NPs) can enter the cell differently depending on their particular size, shape, or mechanical properties, little is known on how the rigidity of NPs affects their entrance into the cell. ${ }^{[1-3]}$ By adopting a microfluidic platform, we synthesized NPs of varying water content and rigidity but with the same chemical composition, size, and surface properties, to show the rigidityregulated cellular uptake of core-shell NPs with a lipid shell rigid or "hard" NPs (less interfacial water between the polymeric core and lipid shell) enter cells more easily than flexible ("soft") ones. Simulations reveal that the "soft" NPs are deformed and thus energetically unfavorable for cellular uptake. Our data therefore pave the way for the design of mechanically favorable NPs for controlled interactions between nanomaterials and cells and better NP-based medical applications.

Lipid-covered polymeric NPs serve well both as a delivery system for drugs-because of their high efficiency and low risk for side effects-and as model systems for understanding the mechanisms of the biological effects of nanomaterials. ${ }^{[4-9]}$ So far, in addition to the chemical composition, size, biocompatibility, and surface properties of the lipid shell of the NPs having influence on the cellular uptake and anticancer efficacy, it has been recognized that different lipid-polymer structures may result in variations in the particle rigidity (the resistance of the particle to deform), thus affecting the cellular uptake of NPs and the efficacy of treatment. ${ }^{[10-12]}$ The link between the rigidity of NPs, and their cellular uptake efficiency, however, is still missing, which is crucial for the design of NPs for drugdelivery. One major challenge of bulk synthesis lies in the

Prof. J. Sun, L. Zhang, Q. Feng, Prof. D. Liu

Prof. B. Ding, Prof. X. Jiang

Beijing Engineering Research Center

for BioNanotechnology \& CAS Key Laboratory

for Biological Effects of Nanomaterials and Nanosafety

National Center for NanoScience and Technology

Beijing 100190, P. R. China

E-mail: xingyujiang@nanoctr.cn

J. Wang, Q. Yin, Prof. Y. Wei, Prof. X. Shi

State Key Laboratory of Nonlinear Mechanics

Institute of Mechanics

Chinese Academy of Sciences

Beijing 100190, P. R. China

E-mail: shixh@imech.ac.cn

Prof. D. Xu

Department of Mechanical and Automation Engineering

The Chinese University of Hong Kong

Shatin, N.T., Hong Kong SAR, China

DOI: 10.1002/adma.201404788 difficulty in the generation of core-shell NPs with a lipid shell containing various amounts of water, which governs the rigidity of the NPs; larger amounts of interfacial water would result in more flexible NPs. ${ }^{[13-15]}$ Microfluidic platforms can generate lipid-polymer hybrid NPs via rapid reaction and precise manipulation of fluids inside microchannels, ${ }^{[16-20]}$ however, the fabrication of hybrid NPs with varying water content has not been achieved by microfluidics. Here, we develop a two-stage microfluidic platform that can assemble core-shell poly(lactic-co-glycolic acid) (PLGA)-lipid NPs in a single-step. ${ }^{[16,21]}$ Lipid-covered PLGA NPs or liposomes that have the same size and surface properties, but varying rigidity as a result of tuning the interfacial water layer, can be realized using the same microchip. It enables us to explore how the rigidity of NPs differentially regulates the cellular uptake and to elucidate the intrinsic mechanism. It also allows the treatment of various diseases through the use of specific particles.

Particle rigidity is tuned by varying the amounts of interfacial water between the PLGA core and lipid shell of the hybrid NPs; this is achieved by altering the injection order of the PLGA and lipid-poly(ethylene glycol) (PEG) organic solutions in the microfluidic chip. The microfluidic device shown in Scheme 1 consists of two stages: 1) The first stage comprises three inlets and a straight synthesis microchannel; 2) The second stage is composed of one centered inlet and a spiral mixing channel (see Supporting Information (SI), Figure S1 for more details). We synthesized particles of varying water content and rigidity using the same chip but different order of the introducing reagents. In mode 1 , the first stage is used for generating PLGA NPs through interfacial precipitation, while the second stage forms lipid-coated NPs as a result of hydrophobic attraction between the lipid tail and PLGA (P-L NPs; Scheme 1A, Figure S2 (SI)). In mode 2, we change the injection order by introducing the lipid solution at the first stage and the PLGA solution at the second stage. In this way, lipids form into a liposome in aqueous solution at the first stage, followed by re-assembly onto the surface of PLGA NPs at the second stage through effective mixing (P-W-L NPs; Scheme 1B, Figure S2 (SI)). The throughput of NPs by a single chip is $41 \mathrm{~mL} \mathrm{~h}^{-1}\left(\approx 8 \mathrm{mg} \mathrm{h}^{-1}\right.$ for $\mathrm{P}-\mathrm{W}-\mathrm{L}$ NPs, and $\approx 6.5 \mathrm{mg} \mathrm{h}^{-1}$ for P-L NPs). For both mode 1 and mode 2 , transmission electron microscopy (TEM) images (Figure 1A; Figure S2, SI) show complete lipid coverage on the surface of PLGA NPs. The different injection order of the solutions may result in the presence of interfacial water between the PLGA core and lipid shell of the P-W-L NPs (mode 2) but not in P-L NPs (mode 1 ), which is confirmed by cryogenic TEM (cryo-TEM Figure 1B; see also SI). For the P-L NPs, the lipid shell is tightly attached to the PLGA core, while for the P-W-L 
A

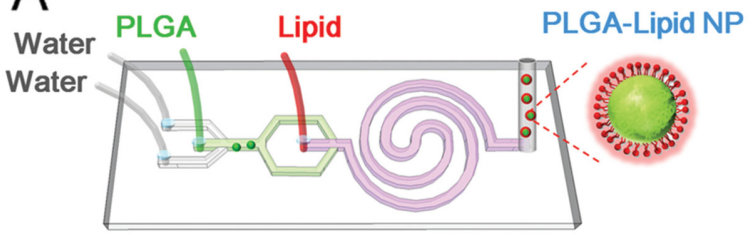

B

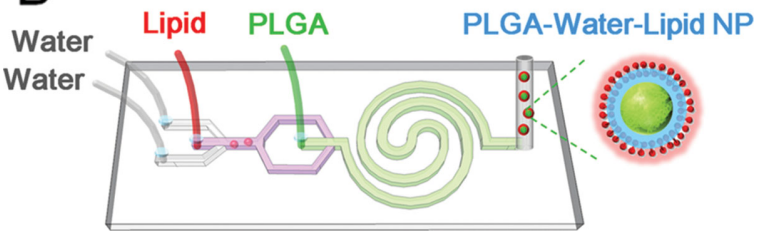

Scheme 1. The two-stage microfluidic platforms for assembling polymerlipid hybrid NPs with varying amounts of water. A) NPs composed of the lipid shell and PLGA core are produced by injecting the PLGA solution in the first stage and lipid-PEC solution in the second stage (mode 1, P-L NPs). B) Nanoparticles composed of the lipid shell, interfacial water layer, and PLGA core are produced by injecting the lipid-PEG solution in the first stage and the PLGA solution in the second stage (mode 2, P-W-L NPs).

NPs, there is a thin water layer between the lipid shell and PLGA core. To estimate the amount of water inside the P-W-L NPs, we measured the radius of the different components of the NPs. The radius of the inner PLGA core is about $27.6 \mathrm{~nm}$, and that of the outer lipid shell is about $31.1 \mathrm{~nm}$. The lipid layer of the P-W-L NP is about $4 \mathrm{~nm}$. Given the radii of the inner and outer spheres, we can calculate the volume of interfacial water to be $3.8 \times 10^{4} \mathrm{~nm}^{3}$, accounting for almost $30 \%$ of the total volume of P-W-L NP. The resolution of the cryo-TEM images obtained with a Tecnai 12 electron microscope appears to be low in comparison to some of the high-resolution protein images, and we may explore it further in future work.

To further characterize the presence of water inside the hybrid NPs generated by two modes of the microfluidic chip, we used Rhodamine $\mathrm{B}(\mathrm{RhB})$ to label the water encapsulated by

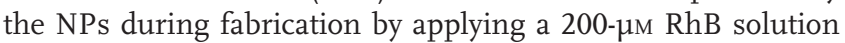
(in water) as the water sheath in both mode 1 and 2 . After synthesis, NPs were separated from free RhB in solution as well as the RhB attached to the surface of the NPs by a Sephadex G50 size-exclusion column (Pharmacia Biotech, NJ, USA), and we concentrated the NPs by evaporating the solution down to $1 \mathrm{~mL}$ with a flow of nitrogen. The fluorescence emission spectrum of the separated NPs was measured at the excitation wavelength of $510 \mathrm{~nm}$ by an LS55 fluorospectrophotometer (Perkin Elmer, MA, USA). The fluorescence intensity of the P-W-L NPs is approximately $50 \%$ higher than that of the P-L NPs (Figure 1C). RhB is a water-soluble dye, and thus most of the RhB encapsulated in the NPs should be in the water layer. There may be a small amount of RhB in the lipid shell and polymer matrix, but this amount should be equal inside the P-L NPs and P-W-L NPs. As a result, the higher fluorescence of P-W-L NPs can only be from the RhB that is encapsulated inside the interfacial water layer.

The existence of more interfacial water inside P-W-L NPs than P-L NPs is associated with the manufacturing

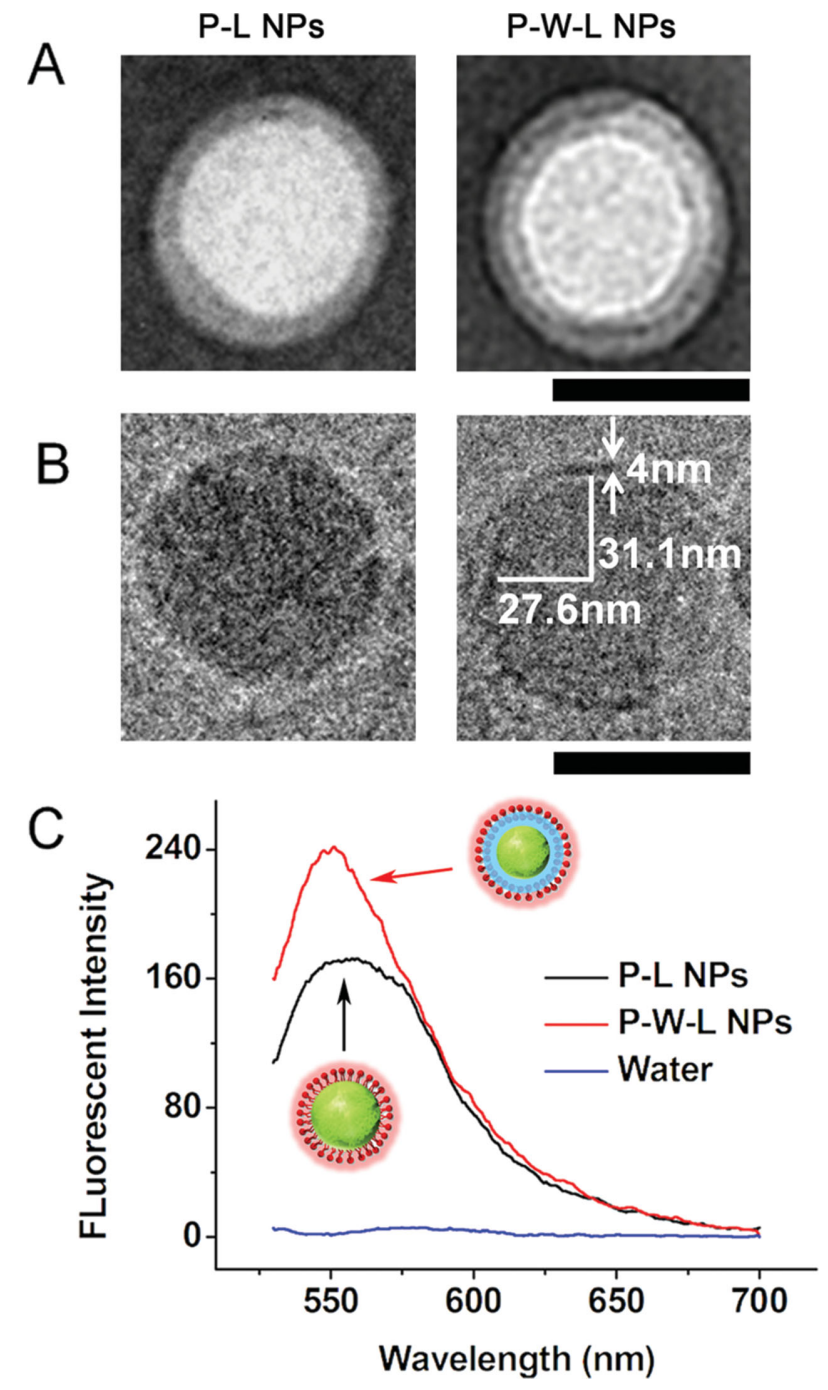

www.MaterialsViews.com

Figure 1. Characterization of the presence of the interfacial water layer inside the NPs. A) TEM images of a P-L NP (left) and P-W-L NP (right); scale bar $=50 \mathrm{~nm}$. B) Cryo-TEM images of a P-L NP (left) and P-W-L NP (right); scale bar $=50 \mathrm{~nm}$. The volume of water content inside the $\mathrm{P}-\mathrm{W}-\mathrm{L}$ NP can be calculated given the radius of the inner PLGA core and that of the outer lipid shell. C) The fluorescence emission spectrum of RhBlabeled NPs indicates that P-W-L NPs contain more water than P-L NPs.

mechanisms of the two-stage microfluidic device, and we used molecular dynamics (MD) simulations to reproduce the process of formation of hybrid NPs (more details in the SI). In mode 1 , when lipids are injected at the second stage, they quickly assemble onto the PLGA NPs before forming liposomes due to the strong hydrophobic interaction between the lipid tail and PLGA (Figure 2A, where lipids are red and blue while PLGA is green). The formation of P-L NPs is indicated by the full coverage of the lipid layer (Figure 2B). In mode 2, since the lipids have already formed liposomes at the first stage, the PLGA solution injected at the second stage and the resultant PLGA NPs cannot enter the liposome unless they are assisted by the vortex in the spiral mixing channel. Figure $2 \mathrm{C}$ shows the process of the PLGA NP contacting the liposome, breaking the membrane of the liposome and finally becoming encapsulated 

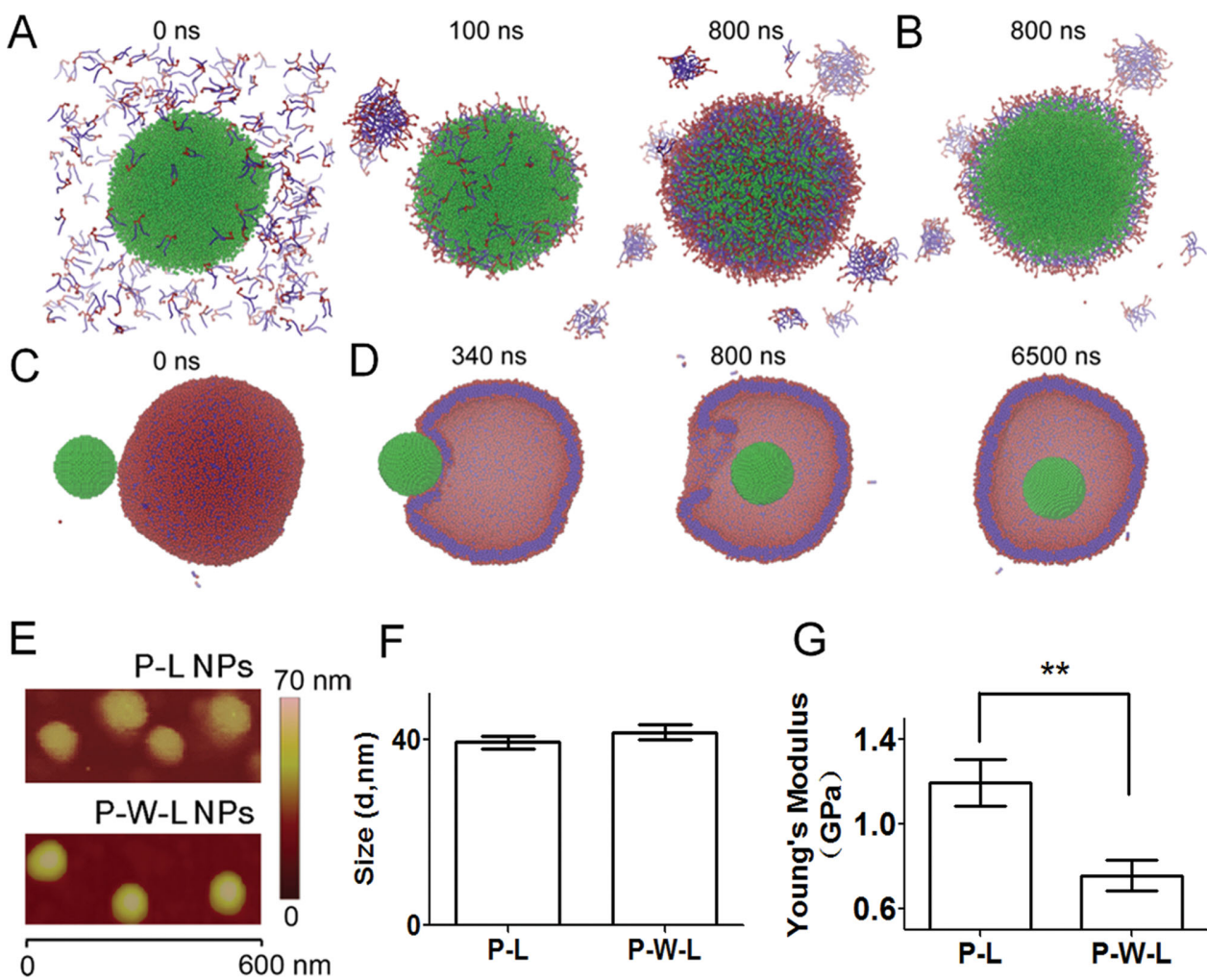

Figure 2. Molecular dynamics simulation and AFM experiments to show lipid-covered NPs (P-L NPs and P-W-L NPs) with varying amounts of water and thus varying rigidity. A) The lipids (colored as red and blue) assemble on the surface of the PLGA NPs (green), resulting in the lipid shell, corresponding to the procedure shown in 1A. B) A slice of the final system (P-L NP) showing the PLCA-lipid structure without the interfacial water layer. C) A vesicle (red and blue) impacted by the PLGA NP (green), in order to form the lipid shell. D) The slices at different time lapses showing the formation of a P-W-L NP with a lipid shell, interfacial water layer, and PLGA core structure, corresponding to the procedure shown in 1B. E) AFM images of P-L NPs and P-W-L NPs. F) Diameter of core-shell NPs from AFM scanning. G) The Young's modulus of P-L NPs (without interfacial water layer) and P-W-L NPs (with interfacial water layer) (**'statistical $P$-value: $P<0.01)$.

in the PLGA NP. In this case, water molecules fill between the lipid shell and PLGA to form the core-water-shell structure of the P-W-L NPs (Figure 2D). We emphasize that this P-W-L NP structure only forms if we have the spiral structures at the second stage. It fails to form in straight channels of the same cross-section and while using the same flow rates. We only observe PLGA NPs and liposomes rather than the core-shell PLGA-lipid NPs in the latter, probably due to inefficient mixing inside the straight channel at the second stage (Figure S3, SI).

The different order of injection and the possible subsequent generation of the interfacial water layer in P-W-L NPs and water-free P-L NPs would give rise to a distinct rigidity for each of the particle types. To validate, we measured the rigidity of P-L and P-W-L NPs usng atomic force microscopy (AFM) with a Multimode 8 Atomic Force Microscope (Bruker) and Nanoscope analysis software (Bruker). For P-L and P-W-L NPs with a size of $40 \mathrm{~nm}$, the Young's modulus of the P-L NPs is
$1.20 \pm 0.11 \mathrm{GPa}$, which is higher than that of the P-W-L NPs $(0.76 \pm 0.07 \mathrm{GPa})$ (Figure $2 \mathrm{E}-\mathrm{G}$, Table 1$)$. The Young's modulus of the P-L NPs is close to that of pure PLGA NPs, which is approximately $1 \mathrm{GPa},{ }^{[22]}$ and three orders of magnitude higher than that of liposomes. ${ }^{[23]}$ We further note that both the P-L NPs and P-W-L NPs exhibit much better long-term stability than the hydrophobic PLGA NPs (without shell) and hollow liposomes (without core) (Figure S4, SI).

Since lipid-covered NPs with distinct rigidity are available, we tested their cellular uptake by incubating the lipid-covered NPs and liposomes with HeLa cells (a type of cancer cell) stained with Hoechst 33342 (blue). We show in Figure 3A-C the results of the cellular uptake of "hard" P-L NPs and "soft" P-W-L NPs. For better visualization, we used DiD (red) and TopFluor-PE (green) to label the PLGA core and lipid shell, respectively. After a 5-h incubation, HeLa cells incubated with P-L NPs display higher green (lipid shell) and red (PLGA core)

Table 1. Properties of $\mathrm{P}-\mathrm{L}$ and $\mathrm{P}-\mathrm{W}-\mathrm{L}$ NPs.

\begin{tabular}{|c|c|c|c|c|c|c|c|}
\hline Structure & Size $[\mathrm{nm}]$ & Surface modification & Surface charge & $\mathrm{EE}^{\mathrm{a})}(\mathrm{DOX})$ & $\mathrm{EE}^{\mathrm{a})}(\mathrm{CA} 4)$ & Internalization pathway & Young's modulus[GPa] \\
\hline PLGA-lipid & $39.5 \pm 1.4$ & PEG & $\approx 0$ & $98.0 \%$ & $90.8 \%$ & $\mathrm{CME}^{\mathrm{b})}$ & $1.20 \pm 0.11$ \\
\hline PLGA-water-lipid & $41.6 \pm 1.6$ & PEG & $\approx 0$ & $97.3 \%$ & $95.3 \%$ & $\mathrm{CME}^{\mathrm{b})}$ & $0.76 \pm 0.07$ \\
\hline
\end{tabular}

a) EE: Entrapment efficiency; ${ }^{\text {b) } C M E: ~ C l a t h r i n-m e d i a t e d ~ e n d o c y t o s i s . ~}$ 


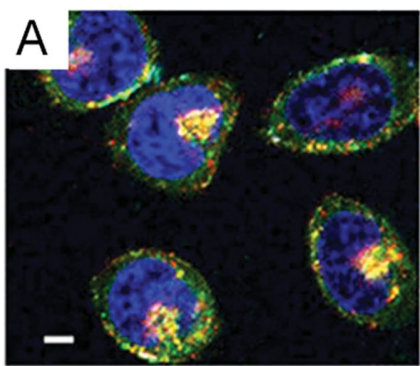

P-L NPs

D

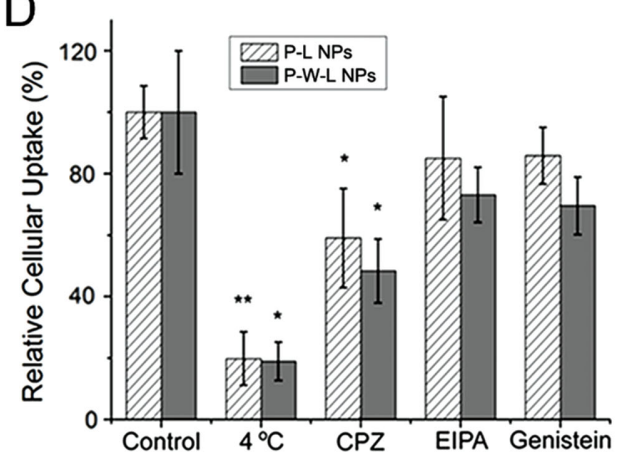

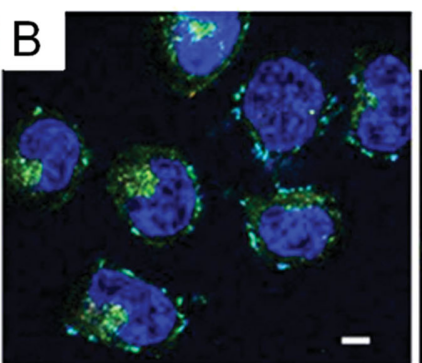

P-W-L NPs

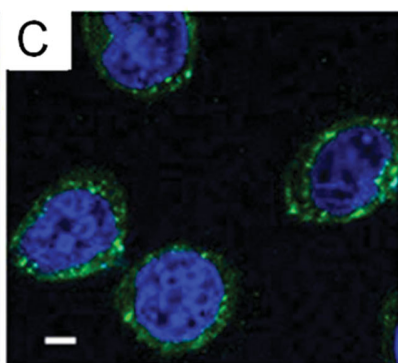

Liposome

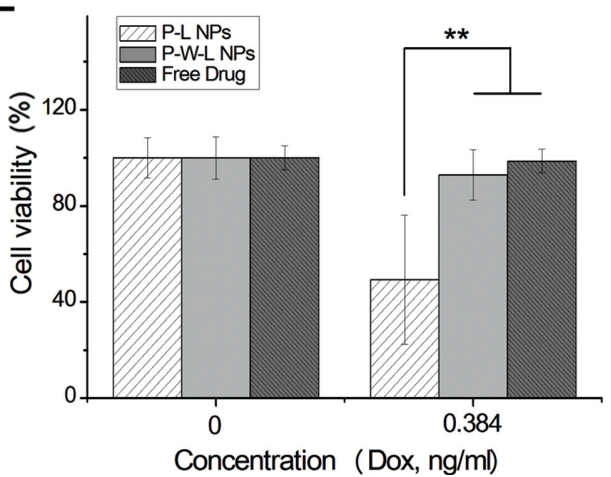

Figure 3. Cellular uptake of NPs (P-L NPs and P-W-L NPs) with varying water content and rigidity. A-C) Confocal fluorescent images of HeLa cells (stained with Hoechst 33342) incubated with P-L NPs (A), P-W-L NPs (B), and liposomes of similar size (C) for 5 h. Co-localization of the PLGA core entrapping DiD (red) and the lipid layer labeled with TopFluo (green) shows a yellow color (scale bar, $5 \mu \mathrm{m})$. D) The effect of hypothermia $\left(4^{\circ} \mathrm{C}\right.$ ) treatment and endocytic inhibitors on the cellular uptake of P-L and P-W-L NPs. The endocytosis inhibitors CPZ, EIPA, and Genistein are used for the inhibition of clathrin-mediated endocytosis, macropinocytosis, and caveolae-mediated endocytosis, respectively. $* P<0.05 ; * * P<0.01$. E) Cell viability quantified by Sulforhodamine B (SRB) assay after a 24-h incubation with P-L NPs, P-W-L NPs, and the free drugs (DOX and CA4). The concentrations of DOX and CA4 are 0 and $0 \mathrm{ng} \mathrm{mL}^{-1}$, respectively, or 0.384 and $1.536 \mathrm{ng} \mathrm{mL}^{-1}$, respectively. $* * P<0.01$.

fluorescence intensities than those incubated with P-W-L NPs (both on the cell membrane and in the cytoplasm), suggesting a significantly higher cellular uptake of the more rigid NPs (P-L NPs) compared with the less rigid ones (P-W-L NPs; Figure 3A, B). HeLa cells incubated with liposomes of similar size have less green fluorescence in the cytoplasm than lipid-covered NPs do (Figure 3C). This rigidity-regulated cellular uptake of NPs was also observed by co-incubation of human umbilical vein endothelial cells (HUVECs) with P-L and P-W-L NPs; HUVECs prefer to take up the more rigid P-L NPs as well (Figure S5, SI). To distinguish the means via which cells internalize these NPs, three endocytosis inhibitors (CPZ for the inhibition of clathrin-mediated endocytosis, EIPA for the inhibition of macropinocytosis, and Genistein for the inhibition of caveolae-mediated endocytosis) and hypothermia treatment $\left(4{ }^{\circ} \mathrm{C}\right.$, inhibition of endocytosis) were applied to HeLa cells before co-incubation with hybrid NPs. ${ }^{[24]}$ HeLa cells treated with $\mathrm{CPZ}$ and hypothermia show reduced uptake of $\mathrm{P}-\mathrm{L}$ and P-W-L NPs, indicating that both NP types are internalized through clathrin-mediated endocytosis (Figure 3D).

To test the ability of these NPs with varying water content and rigidity in cancer treatment, we manufactured hybrid dual-drug-loaded (doxorubicin, DOX, and combretastatin A4, CA4) P-L and P-W-L NPs by adding CA4 into the lipid solution, and DOX into the PLGA solution before microfluidic synthesis. The entrapment efficiency (EE) of DOX and CA4 are $98.0 \%$ and $90.8 \%$, respectively, in $\mathrm{P}-\mathrm{L}$ NPs, and $97.3 \%$ and 95.3\%, respectively, in P-W-L NPs (Figure S6, SI). In-vitro experiments imply that dual-drug-loaded P-L NPs can kill more HeLa cells than both P-W-L NPs encapsulating drugs and free drugs of the same concentrations (DOX: $0.384 \mathrm{ng} \mathrm{mL} \mathrm{m}^{-1}$, CA4: $1.536 \mathrm{ng} \mathrm{mL} \mathrm{m}^{-1}$ ) (Figure 3E). Since the mechanism of in-vivo uptake of NPs is complicated, here we mainly investigate the rigidity-regulated uptake of NPs at the cellular level.

Comparing P-L NPs with P-W-L NPs (including liposomes), the NPs have similar shape, size, composition, surface molecules and internalization pathways (Table 1). The major difference is the rigidity of the NPS related to their internal structure. To understand the mechanism of rigidity-regulated cellular uptake of NPs, we conducted MD simulations to reproduce the internalization process of the NPs into the cell (Figure 4; Movie S1, SI). For the rigid $\mathrm{P}-\mathrm{L} \mathrm{NP}$, it is fully wrapped by the cell membrane and internalized smoothly (Figure 4A). For the less rigid P-W-L NP, however, it undergoes significant deformation during the internalization before it is finally trapped within the cell membrane (Figure 4B). The deformation of the NP impedes its entry into the cell. ${ }^{[1]}$ The combination of particle rigidity with deformation suggests an energy penalty for NPs getting through the cell membrane. To illustrate this concept, we analyzed the elastic deformation energy of the membrane when a spherical NP is very rigid and does not deform, in contrast to the case when the NP can deform to an ellipsoidal shape while entering the cell. Following 
A $0 \mu \mathrm{s}$

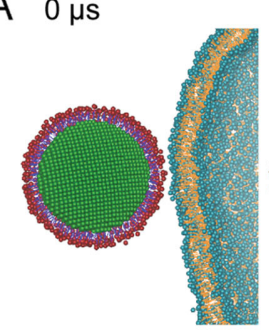

B $0 \mu \mathrm{s}$

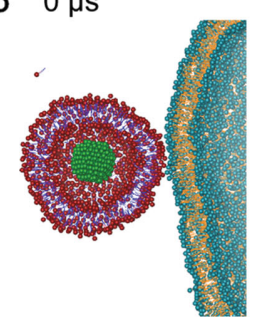

$120 \mu \mathrm{s}$

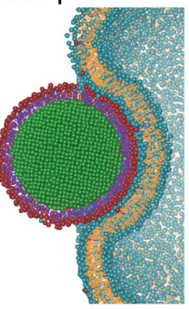

$120 \mu \mathrm{s}$

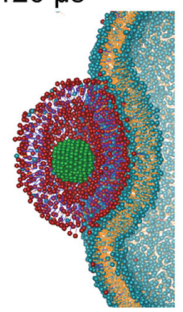

$240 \mu \mathrm{s}$

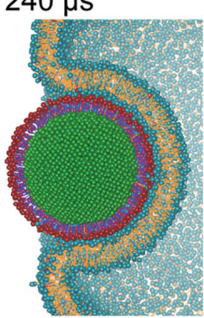

$240 \mu \mathrm{s}$

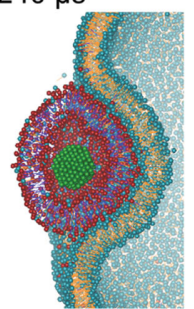

$420 \mu \mathrm{s}$

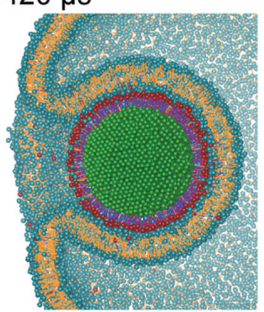

$420 \mu \mathrm{s}$

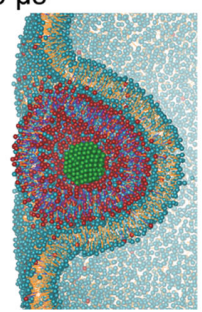

$480 \mu \mathrm{s}$

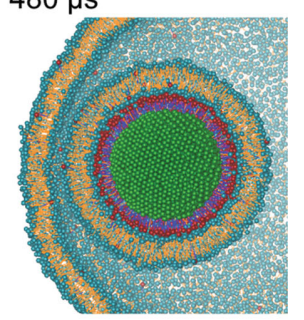

$480 \mu \mathrm{s}$

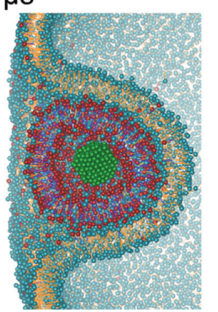

Figure 4. Rigidity-governed deformation influencing cellular uptake as depicted by MD simulations. A) The rigid P-L NP is internalized smoothly via the wrapping of the cellular membrane, showing mild shape deformation. B) The less rigid or "soft" P-W-L NP undergoes large deformation during the internalization, and finally it is trapped on the surface of the cell.

the Canham-Helfrich's framework ${ }^{[25]}$ and considering a spherical NP with a 20-nm radius and an ellipsoidal NP with the same volume and an aspect ratio of $\lambda=0.5$ (where $\lambda$ is defined as a ratio of the minor and major axes of the ellipsoid), we construct a dimensionless parameter $\bar{\sigma}=\sigma K^{2} / \kappa, K$ is the characteristic radius of the NPs and set as $20 \mathrm{~nm}$; the typical membrane bending modulus $\kappa$ is $20 k_{\mathrm{B}} T$ (where $k_{\mathrm{B}} T$ is the thermal energy); and a typical membrane tension $\sigma$ is $0.2 \mathrm{mN} \mathrm{m}^{-1}$. [26] The elastic deformation energy of the membrane is a combination of the bending ( $E_{\text {bending }}$ ) and tension ( $\left.E_{\text {tension }}\right)$ energies; it is expressed as: ${ }^{[25]}$

$$
\begin{aligned}
E_{\text {tot }} & =E_{\text {bending }}+E_{\text {tension }} \\
& =\int \frac{\kappa}{2}\left(c_{1}+c_{2}\right)^{2} d S+\int \sigma(1-\cos \psi) d S
\end{aligned}
$$

where $c_{1}$ and $c_{2}$ are principal curvatures of the membrane surface, $\psi$ is the angle tangent to the membrane profile, and $d S$ are the elements of the membrane surface. Through energy minimization of the total energy, we will obtain the elastic deformation energy of the membrane as a function of the wrapping fraction. ${ }^{[27]}$ The elastic deformation energy as a function of the wrapping fraction for spherical and ellipsoidal NPs reveals that complete wrapping of ellipsoidal NPs requires about $30 \%$ more energy than spherical ones (Figure S7, SI). This may partly explain why the less rigid and more flexible NPs are relatively difficult for cellular uptake. As shown in our MD simulation, the more flexible NPs are able to deform themselves into ellipsoidal NPs where more binding energy is required to overcome the bending energy to complete the internalization (Figure S7, SI). Recent studies indicate that the Young's modulus and stiffness of the less rigid, "soft" hydrogel particles (i.e., poly(methacrylic acid) (PMA) hydrogel particles) could be tuned over an order of magnitude by controlling the cross-linker concentration. ${ }^{[28]}$ Despite the low Young's modulus (less than several mega-Pascals) of the less rigid hydrogel particles compared to the lipid-covered PLGA NPs (i.e., P-L NPs; $\approx 1 \mathrm{GPa}$ ), the hydrogel particles with tunable rigidity remain promising for novel therapeutic applications and the construction of biomimetic models such as the blood capillary. ${ }^{[29]}$

Rigidity is important for biology, ${ }^{[30,31]}$ but we may be the first to demonstrate that it is also important in drug delivery. To the best of our knowledge, this is the only report to test the biological effects of the varying rigidity of nanoscale particles of the same composition, surface chemistry, and size. We extended our understanding by exploring the relationships among the polymer-lipid structure, the rigidity of the NPs for drug delivery, and cellular uptake efficiency. We developed a two-stage microfluidic chip that can be readily scaled up to produce NPs of the same chemical composition, size, and surface properties, but varying amounts of water and rigidity. It enabled us to explore how the rigidity of NPs regulates the cellular uptake and to elucidate the intrinsic mechanism. Given the only significant difference between the NPs we have presented is the rigidity, our experiments suggest that rigidity can dramatically alter the cellular uptake efficiency, with more rigid NPs able to move more easily through membranes. The mechanisms revealed here suggest that tuning the rigidity of NPs is an appealing way to improve therapeutic efficiency. Combined with the experimental approach we have developed, we expect the results presented in this work to pave a new way to design mechanically favorable NPs for better NP-based drug delivery and possibly other medical applications.

\section{Supporting Information}

Supporting Information is available from the Wiley Online Library or from the author.

\section{Acknowledgements}

J.S., L.Z., and J.W. contributed equally to this work. X.J. and J.S. acknowledge financial support from MOST (2013AA032204, and 
2013YQ190467), NSFC (21475028, 51105086, 21025520, and 81361140345), Beijing Municipal Science \& Technology Commission (Z131100002713024), Chinese Academy of Sciences (XDA09030305, XDA09030308), and the CAS/SAFEA International Partnership Program for Creative Research Teams. X.S. acknowledges financial support from NSFC (11422215, 11272327, and 11023001). J.S. thanks Prof. Deyu Li from Vanderbilt University for helpful discussion. The authors thank Dr. Shuai Zhang from Aarhus University for helpful discussion on the AFM measurements.

Received: October 16, 2014 Revised: November 24, 2014 Published online:

[1] F. Ye, C. Wu, Y. Jin, Y. H. Chan, X. Zhang, D. T. Chiu, J. Am. Chem. Soc. 2011, 133, 8146-8149.

[2] M. Schulz, S. Werner, K. Bacia, W. H. Binder, Angew. Chem. Int. Ed. 2013, 52, 1829-1833.

[3] M. Wang, K. Alberti, S. Sun, C. L. Arellano, Q. Xu, Angew. Chem. Int. Ed. 2014, 53, 2893-2898.

[4] A. Schroeder, D. A. Heller, M. M. Winslow, J. E. Dahlman, G. W. Pratt, R. Langer, T. Jacks, D. G. Anderson, Nat. Rev. Cancer 2012, 12, 39-50

[5] T. R. Pearce, K. Shroff, E. Kokkoli, Adv. Mater. 2012, 24, 3803-3822.

[6] J. Yoon, J. Kwag, T. J. Shin, J. Park, Y. M. Lee, Y. Lee, J. Park, J. Heo, C. Joo, T. J. Park, P. J. Yoo, S. Kim, J. Park, Adv. Mater. 2014, 26, $4559-4564$.

[7] J. S. Sun, Y. L. Xianyu, X. Y. Jiang, Chem. Soc. Rev. 2014, 43, 6239-6253.

[8] S. Sengupta, D. Eavarone, I. Capila, G. Zhao, N. Watson, T. Kiziltepe, R. Sasisekharan, Nature 2005, 436, 568-572.

[9] S. Tan, X. Li, Y. Guo, Z. Zhang, Nanoscale 2013, 5, 860-872.

[10] X. H. Shi, A. von dem Bussche, R. H. Hurt, A. B. Kane, H. J. Gao, Nat. Nanotechnol. 2011, 6, 714-719.

[11] X. Yi, X. H. Shi, H. J. Gao, Phys. Rev. Lett. 2011, 107, 098101.

[12] L. Zhang, J. M. Chan, F. X. Gu, J. W. Rhee, A. Z. Wang, A. F. Radovic-Moreno, F. Alexis, R. Langer, O. C. Farokhzad, ACS Nano 2008, 2, 1696-1702.
[13] P. M. Valencia, O. C. Farokhzad, R. Karnik, R. Langer, Nat. Nanotechnol. 2012, 7, 623-629.

[14] J. M. Chan, L. Zhang, K. P. Yuet, G. Liao, J. W. Rhee, R. Langer, O. C. Farokhzad, Biomaterials 2009, 30, 1627-1634.

[15] Y. Kim, B. Lee Chung, M. Ma, W. J. Mulder, Z. A. Fayad, O. C. Farokhzad, R. Langer, Nano Lett. 2012, 12, 3587-3591.

[16] J. Sun, Y. Xianyu, M. Li, W. Liu, L. Zhang, D. Liu, C. Liu, G. Hu, $X$. Jiang, Nanoscale 2013, 5, 5262-5265.

[17] Y. Zhang, Y. Guo, Y. Xianyu, W. Chen, Y. Zhao, X. Jiang, Adv. Mater. 2013, 25, 3802-3819.

[18] B. Yuan, Y. Jin, Y. Sun, D. Wang, J. Sun, Z. Wang, W. Zhang, X. Jiang, Adv. Mater. 2012, 24, 890-896.

[19] J. Wang, W. Chen, J. Sun, C. Liu, Q. Yin, L. Zhang, Y. Xianyu, X. Shi, G. Hu, X. Jiang, Lab Chip 2014, 14, 1673-1677.

[20] H. C. Shum, J. W. Kim, D. A. Weitz, J. Am. Chem. Soc. 2008, 130, 9543-9549.

[21] Q. Feng, M. Z. Yu, J. C. Wang, W. J. Hou, L. Y. Gao, X. F. Ma, X. W. Pei, Y. J. Niu, X. Y. Liu, C. Qiu, W. H. Pang, L. L. Du, Q. Zhang, Biomaterials 2014, 35, 5028-5038.

[22] J. H. Park, M. G. Allen, M. R. Prausnitz, J. Controlled Release 2005, 104, 51-66.

[23] X. M. Liang, G. Z. Mao, K. Y. S. Ng, J. Colloid Interface Sci. 2004, $278,53-62$.

[24] J. Sun, M. Li, C. Liu, Y. Zhang, D. Liu, W. Liu, G. Hu, X. Jiang, Lab Chip 2012, 12, 3952-3960.

[25] M. Deserno, Phys. Rev. E 2004, 69, 031903.

[26] C. E. Morris, U. Homann, J. Membr. Biol. 2001, 179, 79-102.

[27] S. Dasgupta, T. Auth, G. Gompper, Soft Matter 2013, 9, 54735482 .

[28] J. P. Best, J. W. Cui, M. Mullner, F. Caruso, Langmuir 2013, 29, 9824-9831.

[29] J. Cui, M. Bjornmalm, K. Liang, C. Xu, J. P. Best, X. Zhang, F. Caruso, Adv. Mater. 2014, 26, 7295-7299.

[30] J. Fu, Y. K. Wang, M. T. Yang, R. A. Desai, X. Yu, Z. Liu, C. S. Chen, Nat. Methods 2010, 7, 733-736.

[31] Y. Sun, K. M. Yong, L. G. Villa-Diaz, X. Zhang, W. Chen, R. Philson, S. Weng, H. Xu, P. H. Krebsbach, J. Fu, Nat. Mater. 2014, 13, 599-604. 